\title{
Investing in Endovascular Thrombectomy Programs: Saving Lives, Saving Money
}

Keywords: Stroke, Costs, Economics, Endovascular therapy, Cost-benefit analysis

In investing, what is comfortable is rarely profitable. - Robert Arnott

Stroke is one of the leading causes of mortality and morbidity worldwide, leaving approximately one in four stroke survivors with significant disability. ${ }^{1,2}$ In addition, stroke care is costly, with Canada alone spending approximately 3.6 billion dollars per year in stroke care. ${ }^{3}$ These costs include both direct costs (i.e., hospital beds, hospital personnel and time, diagnostic imaging, surgical interventions, prescription medications, and physician costs) and indirect costs (i.e., out-of-pocket expenses for rehabilitation, loss of productivity, and informal caregiving). ${ }^{4}$ Use of catheter-based endovascular thrombectomy (EVT) in eligible patients has been associated with a significant reduction in the disability and mortality associated with ischemic stroke. ${ }^{3}$ However, EVT is only available for patients with an ischemic stroke due to a large-vessel arterial occlusion, approximately $25-46 \%$ of all acute ischemic stroke patients, ${ }^{5}$ and of these, only few meet the inclusion criteria for EVT based on severity of symptoms and brain imaging. Further, it has additional costs associated with inter-hospital transfers; procedural and post-procedural care; and personnel costs, and so, it is only available at select few centers. ${ }^{6}$ Therefore, at a population level, investing in EVT programs that benefit only a selected few has been an area of considerable debate for policy makers and health economists.

In this issue of the Journal, Thanh et al. evaluate if investments in provincial EVT programs can help save money from a provincial perspective. ${ }^{7}$ They examined the cost benefit of a provincial strategy, Endovascular Reperfusion Alberta (ERA) project, that aimed to improve access to EVT in Alberta. This provincially funded strategy included investments of \$2.04 million and \$3.73 million in the years 2018 and 2019, respectively, in the following areas: revision of emergency medical services triage and transport pathways, inter-hospital referrals, establishment of imaging in remote stroke centers, and improvement in processes to reduce treatment times. Subsequently, 172 and 218 more people received EVT in 2018 and 2019, respectively. To evaluate the savings that may have occurred from increasing access to EVT, the authors used health service utilization data and the associated direct costs, obtained from standardized provincial health care cost data, of 99 patients (52 of whom received EVT) with acute ischemic stroke who were enrolled in the $\mathrm{ESCAPE}^{8}$ randomized controlled trial in 2013-2014. The savings were calculated as a function of costs avoided by the reduced health care utilization among those who received EVT compared to those who did not. These were inflated to 2019 Canadian dollars. The net benefit was calculated as the sum of savings (from costavoidance) for all additional patients receiving EVT minus the provincial investment in the year. Overall, the greatest net cost saving was found at 1 -year $(\$ 54,592$ per patient) and 5-year (\$47,070 per patient) time-points, but not at 90 days $(-\$ 7,313$ per patient). At a population level, this translates to an estimated net savings of \$9.4-\$8.11 million in the long term, and a return on investment (ROI), calculated as the ratio of benefit and cost, of 5-5.6. The provincial strategy was cost beneficial across various sensitivity analyses. These findings mirror those of others that suggest EVT is cost-effective. ${ }^{6,9}$

It is impossible to directly attribute the increase in the number of people who received EVT to the provincial strategy, but such ecological fallacy ${ }^{10}$ plagues all public health interventions unless they are studied in natural experiment studies ${ }^{11}$ or cluster randomized trials. ${ }^{12}$ Unfortunately, cluster randomized trials are not possible for all new life-saving interventions due to the costs associated with such trials or the lack of clinical equipoise. Another important limitation acknowledged by the authors is the lack of data on indirect costs in their analysis. While some of these may not fall under the mandate of the provincial health ministry, a cross ministerial committee (including finance and health) could help make an economic argument for investment in EVT programs, especially because it could help reduce lost productivity in young stroke survivors. ${ }^{13}$ A review of ROIs from 23 public health interventions in the UK found the median ROI of any public health intervention to be $14.3,{ }^{14}$ placing the ROI from the ERA project below the median. However, the ROI of local public health interventions is considerably lower (median ROI 4.1) compared to national public health interventions (median ROI 27.2), ${ }^{14}$ supporting the need to develop a national coordinated stroke strategy to improve EVT access and delivery.

The study by Thanh et al. ${ }^{7}$ highlights the importance of longterm follow-up when evaluating public health interventions. For episodic conditions such as stroke, where the costs of stroke care are significant in the first few days following stroke, it is easy to conclude that an intervention has no benefit (or is harmful) if it is 
evaluated only in the short term. Further, public health interventions often have high start-up costs (e.g., setting up an EVT center), but low operating costs, reaping long-term returns on upfront investments. Lastly, this study underscores the value of linking clinical trial data to robust administrative databases for long-term evaluation. ${ }^{15}$

Some unanswered questions remain: What components of the ERA project are most important in reducing the costs? Could similar provincial strategies be effective in other jurisdictions where more than just two EVT centers may be present, or with a different geographical landscape? Lastly, can the economic argument of a high ROI help mobilize scarce healthcare resources to deliver guideline-recommended life-saving therapies to larger populations?

In summary, despite the considerable upfront investments required to improve EVT access and care, it saves lives at a population level and this study shows that it can help save money too, especially in the long term. These savings can (or should) be potentially used to improve access to EVT in remote areas, making it equitable at a population level, and to improve stroke prevention and acute stroke care for non-EVT-eligible patients.

\section{ACKNOWLEDGEMENTS}

We thank Dr Amy Y.X. Yu and Dr Moira K. Kapral for their helpful comments on a previous version of this manuscript.

\section{DisClosures}

The authors have no conflicts of interest.

\section{Statement of Authorship}

AMK and MVV were in conception of the study and co-wrote the manuscript. MVV supervised AMK and is the guarantor.

Andrea M. Kuczynski Division of Neurology, University of Toronto, Toronto, Canada

Manav V. Vyas (iD Division of Neurology, University of Toronto, Toronto, Canada Li Ka Shing Knowledge Institute, St. Michael's Hospital-Unity Health Toronto, Toronto, Canada
Correspondence to: Manav V. Vyas, Clinician-Scientist, St. Michael's Hospital of Unity Health Toronto, Division of Neurology, University of Toronto, Stroke \&Neurology Clinic, 6th

Floor, 30 Bond Street, Toronto, Ontario M5B 1W8, Canada. Email: manav.vyas@mail.utoronto.ca

\section{REFERENCES}

1. Donnan GA, Fisher M, Macleod M, Davis SM. Stroke. Lancet. 2008;371:1612-23.

2. Patel MD, Tilling K, Lawrence E, Rudd AG, Wolfe CDA, McKevitt C. Relationships between long-term stroke disability, handicap and health-related quality of life. Age Ageing. 2006;35:273-9.

3. Boulanger JM, Lindsay MP, Gubitz G, et al. Canadian stroke best practice recommendations for acute stroke management: prehospital, emergency department, and acute inpatient stroke care, 6th edition, update 2018. Int J Stroke. 2018;13:949-84.

4. Mittmann N, Seung SJ, Hill MD, et al. Impact of disability status on ischemic stroke costs in Canada in the first year. Can J Neurol Sci. 2012;39:793-800.

5. Rennert RC, Wali AR, Steinberg JA, et al. Epidemiology natural history, and clinical presentation of large vessel ischemic stroke. Neurosurgery. 2019;85:S4-S8.

6. Kunz WG, Almekhlafi MA, Menon BK, et al. Public health and cost benefits of successful reperfusion after thrombectomy for stroke. Stroke. 2020;51:899-907.

7. Thanh N, Jeerakathil T, Stang J, et al. Return on investment in endovascular care: the case of endovascular reperfusion Alberta. Can J Neurol Sci. 2021;49:629-35.

8. Goyal M, Demchuk AM, Menon BK, et al. Randomized assessment of rapid endovascular treatment of ischemic stroke. N Engl J Med. 2015;372:1019-30.

9. Xie X, Lambrinos A, Chan B, et al. Mechanical thrombectomy in patients with acute ischemic stroke: a cost-utility analysis. CMAJ Open. 2016;4:E316-325.

10. Pearce N. The ecological fallacy strikes back. J Epidemiol Community Health. 2000;54:326-7.

11. Vyas MV, Fang J, Kapral MK. Temporal trends in the unmet health care needs of Canadian stroke survivors. Can J Neurol Sci. 2020;47:176-82.

12. Hemming K, Eldridge S, Forbes G, Weijer C, Taljaard M. How to design efficient cluster randomised trials. BMJ. 2017;358: j3064.

13. Vyas MV, Hackam DG, Silver FL, Laporte A, Kapral MK. Lost productivity in stroke survivors: an econometrics analysis. Neuroepidemiology. 2016;47:164-70.

14. Masters R, Anwar E, Collins B, Cookson R, Capewell S. Return on investment of public health interventions: a systematic review. J Epidemiol Community Health. 2017;71:827-34.

15. Henry D, Fitzpatrick T. Liberating the data from clinical trials. BMJ. 2015;351:h4601. 